\title{
Análisis del desarrollo humano relativo al género en la región norte de México, 1995-2005
}

\author{
Francisco José Zamudio Sánchez* \\ Montserrath Serrano Morales** \\ María del Rosario Ayala Carrillo ${ }^{* * *}$
}

Resumen: El desarrollo humano es la expresión de un proceso por medio del cual se ofrecen oportunidades a hombres y mujeres para vivir más, educarse y contar con ingresos que se reflejen en una vida mejor. Sin embargo, tales oportunidades se han negado históricamente a la mujer, así como su participación en actividades económicas, políticas y de toma de decisiones, por ello, el objetivo de este artículo es dar a conocer el grado de desarrollo y empoderamiento femeninos respecto de los masculinos, en la región norte de México (Coahuila, Chihuahua y Durango). Los resultados indican que las mujeres sólo lograron aminorar la inequidad en las opciones para desarrollar sus capacidades, mas no para usarlas.

Palabras clave: desarrollo, empoderamiento, género, inequidad, mujeres.

\footnotetext{
* Profesor-investigador, Departamento de Estadística, Matemática y Cómputo (IDEMyc), Universidad Autónoma Chapingo. Km 38.5 carretera México-Texcoco, C. P. 56230, Chapingo, México.Teléfono (595) 954 7078. Correo electrónico: fjzams@yahoo.com

** Licenciada en estadística por la Universidad Autónoma Chapingo. Av. 20 de Noviembre \#10, San Rafael Tenanyecac, Nativitas, Tlaxcala, México. Teléfono (246) 4160796 . Correo electrónico: basemo23@yahoo.com.mx

${ }^{* * * *}$ Maestra en ciencias del desarrollo rural por el Colegio de Postgraduados. Calle Texas \# 8, San Luis Huexotla, Texcoco, Estado de México. Teléfono (595) 928 5119. Correo electrónico: rosarioa_12@yahoo.com.mx
} 
Abstract: Human development is the expression of a process through which men and women are offered opportunities to have a longer life, more education and a greater income, which lead to improved well-being. However, such opportunities have been historically denied to women, as has been their participation in economic, political and decision-making activities. The objective of this article is to show the level of development and empowerment for women versus men in the northern region of Mexico (Coahuila, Chihuahua and Durango). The results show that women have reduced the gap in the options they have for developing their capacities, though inequity in their usage persists.

Key words: development, empowerment, gender, inequity, women.

\section{Introducción}

El género es un principio clave para organizar la distribución del trabajo, propiedad y otros recursos de la sociedad. Las relaciones desiguales de género se sostienen y legitiman a través de ideas sobre diferencias, que expresan creencias y valores muy extendidos sobre la "naturaleza" de la masculinidad y feminidad. Las normas, costumbres y prácticas institucionalizadas ayudan a explicar la distribución de recursos y responsabilidades de género en los grupos sociales (Kabeer 2006, 76).

El origen de las desigualdades e inequidades de género ha sido la división tradicional del trabajo, que determina la posición de hombres y mujeres en la esfera de lo productivo/público y reproductivo/doméstico/privado respectivamente. Las formas en que una mujer se relaciona con otros u otras depende de las construcciones culturales atribuidas a su género, por lo que el acceso o exclusión a ciertos estatus y proyectos son diferentes a los de los hombres (Teyssier 1997, 179).

Las actitudes, creencias y prácticas utilizadas para excluir a las mujeres están profundamente arraigadas y, en muchos casos, aso- 
ciadas con normas culturales, sociales y religiosas. En México, como en el mundo, se deja al descubierto la discriminación de género cuando a las mujeres se les reducen las oportunidades de generación de ingresos, acceso a la salud y educación. El desarrollo de un país no sólo implica el progreso material e ingreso económico, sino también que las personas tengan igualdad de oportunidades para conseguir sus objetivos, libertad para ejercer sus derechos y toma de decisiones a lo largo de su vida, así se concibe desde el enfoque del desarrollo humano (DH).

En los estudios del desarrollo, el género no fue tomado en cuenta por las estrategias orientadas al crecimiento, pues éstas no solían considerar el "factor humano". En la década de 1970 se empezó a prestar mayor atención a las necesidades básicas, la productividad rural y a las actividades del sector informal, hubo entonces un interés creciente por el papel de la mujer en el desarrollo, que se expresó de dos formas: a) un argumento en favor de la equidad económica y b) la representación de la mujer como el ser más pobre entre los pobres. Ambas partieron de la premisa de que ellas son un factor económico importante (Kabeer 2006, 27).

El concepto de desarrollo humano se ha basado sobre todo en la propuesta de Amartya Sen; surge como una forma de poner en práctica el desarrollo teórico de las capacidades y realizaciones planteadas por Sen (1997). El enfoque de las capacidades constituye el fundamento teórico y permite relacionar el DH y la inequidad de género. Supone la creación de un entorno en el que las personas puedan vivir en forma productiva y creativa de acuerdo con sus necesidades e intereses. Para Sen, no son las necesidades básicas, los bienes primarios o los recursos con que se cuenta, sino las capacidades las que permiten llegar a hacer, ser, lograr y desarrollar las habilidades para alcanzar aquello que se valora.

El enfoque de las capacidades implica que los planificadores sociales investiguen y atiendan las necesidades de la gente, los recursos con que cuenta y las habilidades para convertirlos en funcionamientos. Por lo tanto, lograr el desarrollo de las capacidades es un elemento fundamental para el diseño de las políticas, programas y acciones del sector público, medir avances o retrocesos en las condiciones de vida de sus habitantes, establecer la magnitud del proble- 
ma del desarrollo, caracterizar el fenómeno y definir con claridad los objetivos que se persiguen en términos de bienestar (LópezCalva et al. 2004, 2).

Sin embargo, para mejorar las condiciones sociales, económicas y políticas del país es necesario incorporar y hacer valer la igualdad de género y el empoderamiento de las mujeres. Para lograrlo, es indispensable reconocer que mujeres y hombres desempeñan papeles y responsabilidades distintos en sus propias vidas, dentro de sus familias, en su comunidad y en la sociedad, sin perder de vista que esas diferencias no deben traducirse en discriminación.

De acuerdo con Sen (1997), la pertenencia a un sexo otorga socialmente facultades distintas, asimétricas, para construir libertades esenciales, aun cuando se disponga de bienes primarios y capacidades iguales. Desde esta concepción del desarrollo, es necesario tomar en cuenta las necesidades de las mujeres como fuerza productiva, y analizar desde una perspectiva de género las políticas que se implantan.

Martha Nussbaum $(1999,5)$ en su enfoque de capacidades (desde la teoría feminista) considera que "todos, sólo por ser humanos, son de igual dignidad y valor", por lo que se debe respetar y promover la igualdad de las personas. Para ello es necesario analizar las preferencias y necesidades de hombres y mujeres de manera diferenciada, pues no se puede hablar de desarrollo humano si se impiden o escatiman las libertades sustanciales de las mujeres.

El paradigma del DH establece que la igualdad de oportunidades es ser o hacer aquello que una persona valora para su propia vida como fundamental. Sin embargo, si hay restricciones para elegir las mejores opciones (principalmente para las mujeres) será imposible avanzar hacia un DH pleno del país, porque la mitad de la población no tiene esa posibilidad (Programa de las Naciones Unidas para el Desarrollo, PNUD 2009, 25).

Según el Informe de Desarrollo Humano 1995 (PNUD 1995, 35), en ninguna sociedad las mujeres disfrutan de las mismas oportunidades que los hombres, por lo que incorporar la perspectiva de género al enfoque del DH permite identificar las causas y factores que impiden, obstaculizan o promueven el ejercicio pleno de los derechos de ellas, así como la igualdad y la no discriminación (Ibid. 2006, 21). 
De los esfuerzos realizados en el mundo para medir de manera sistemática el DH, quizá el más destacado sea el índice de desarrollo humano (IDH), propuesto por el PNUD, que en los últimos 19 años ha estudiado la medida del desarrollo humano en más de 170 países. Para ello, ha promovido la elaboración teórica, metodológica e investigación sistemática con las que ha diseñado el IDH, que permite clasificar a las naciones según el grado alcanzado por las capacidades humanas básicas.

En el presente artículo se analizan tres IDH:

- Índice de desarrollo humano con producto interno bruto (idhp), en dólares de paridad de poder adquisitivo (PPA) ${ }^{1}$

- Índice de desarrollo relativo al género (idg)

- Índice de empoderamiento de género (ipg)

El idhp mide las capacidades alcanzadas y opciones disponibles que las personas tienen para desarrollarlas, tomando en cuenta la esperanza de vida como indicador de salud, educación como expresión de capital humano e ingreso que haga posible una vida digna, que sintetizan indicadores complejos que no abarcan sólo al producto nacional bruto ni se centran en él. El propósito del idg es reflejar la inequidad de género en los mismos componentes básicos contenidos en el idhp. El ipg se concentra en la participación económica, política y profesional, y difiere del idg, que se refiere a las capacidades básicas y niveles de vida. Al igual que el idhp y el idg, el ipg se apoya en algunas variables escogidas: el ingreso medido por el producto interno bruto per cápita Pibp en dólares PPA, la participación en empleos clasificados como profesionales y técnicos o administrativos y ejecutivos y la proporción de escaños parlamentarios.

En el presente artículo, enfocado en la región norte de México, se plantea la importancia de estudiar el desarrollo y empoderamiento

\footnotetext{
${ }^{1}$ Las tasas de PPA permiten determinar el número de unidades de la moneda de un país, necesarias para adquirir la misma canasta representativa de bienes y servicios que un dólar estadounidense adquiriría en Estados Unidos de América. La población que recibe un ingreso per cápita por debajo de un dólar PPA al día, significa que vive con menos de 1.08 dólares diarios.
} 
de género, ya que su colindancia con Estados Unidos ha inducido un crecimiento económico en la zona y como consecuencia en la equidad de género, por lo que es considerada estratégica para el progreso nacional. Según el Programa para la Frontera Norte, dicha región destaca como una de las de mayor dinamismo en todo el país. El gran crecimiento demográfico e industrial, los contrastes sociales, económicos y políticos, la interdependencia de las ciudades mexicanas con las de Estados Unidos y sus características geográficas la hacen prioritaria para el diseño de políticas públicas y programas de gobierno (Secretaría de Medio Ambiente y Recursos Naturales, SEMARNAT 2008).

Uno de los objetivos de este artículo es mostrar que la inequidad de género está presente en la vida cotidiana de todas las personas, en especial de las mujeres y en específico en las de Coahuila, Chihuahua y Durango, estados que conforman la región norte de México según el geógrafo mexicano Ángel Bassols Batalla (1999), con base en sus características geográficas, socioeconómicas y culturales similares.

\section{Metodología}

Los indicadores obtenidos para medir la situación de inequidad de las mujeres fueron el idg y el ipg, siguiendo la metodología del PNUD.

Para elaborar los informes de México sobre DH, 1995, 2000 y 2005, los indicadores que se construyen con cada variable se hacen del siguiente modo: ${ }^{2}$

donde:

$$
\text { In }=\frac{\text { valor }- \text { mín }}{\text { máx }- \text { mín }}
$$

valor: es el tomado por la variable correspondiente

mín: es el valor mínimo de la variable correspondiente

máx: es el valor máximo de la variable correspondiente.

\footnotetext{
${ }^{2}$ Para ver con detalle la construcción de los indicadores e índices, consultar la memoria técnica en la página http://www.chapingo.mx/dicifo/demyc/idh
} 
Cuando la variable se trata del Pibp, éste se transforma por medio del logaritmo natural.

La escala de estudio es la unidad básica de la división política de la república mexicana, es decir, la municipal. De cada variable estudiada en los municipios se obtiene un indicador y con ellos se construyen los índices de desarrollo correspondientes. Se analizaron 2 428, 2443 y 2454 municipios, que son los reportados por el Instituto Nacional de Estadística, Geografía e Informática (INEGI) para 1995, 2000 y 2005 respectivamente, más los 32 estados y el del país.

Índice de desarrollo humano con producto interno bruto

Este índice considera al Pibp en dólares $\mathrm{PPA}^{3}$ como el factor de ingreso que representa la parte del DH que no está en la salud ni en la educación. En la construcción de este índice se utilizan cuatro variables: esperanza de vida, tasa de alfabetización, niños de 6 a 14 años que saben leer y escribir (aproximación de la matrícula infantil) y el Pibp en dólares PPA.

El idhp se calculó con la fórmula:

$$
i d h p=\frac{\ln E s p+\left(\frac{2}{3} \operatorname{In} A l f+\frac{1}{3} \ln M a t\right)+\operatorname{In} P i b p}{3}
$$

donde los indicadores son:

InEsp: esperanza de vida

InAlf: alfabetización

InMat: matriculación infantil

InPibp: producto interno bruto per cápita

\footnotetext{
${ }^{3}$ Método para transformar el Pibp en dólares PPA a pesos de 1993: a) El Pib per cápita en dólares PPA se multiplica por el factor de conversión (PPA 1996) $=6.14537906646729$; b) el valor obtenido se divide por $\mathrm{k}=3.68363804420555$, esto para llevarlo a pesos de 1993 .
} 
La expresión entre paréntesis es el índice de educación (InEdu), la combinación de los indicadores de alfabetización y de matriculación.

Los máximos y mínimos para cada variable involucrada en los cálculos de los indicadores del idhp para 1995, 2000 y 2005 están en la figura 1.

\section{Figura 1}

Máximos y mínimos de cada variable para el cálculo del idhp

\begin{tabular}{|l|r|r|}
\hline Variable & Máximo & Mínimo \\
\hline Esperanza de vida & 90 & 30 \\
\hline Alfabetización & 100 & 0 \\
\hline Niños de 6 a 14 años & 100 & 0 \\
\hline Ingreso (PPA en dólares estadounidenses) & $\operatorname{Ln}^{*}(40000)$ & $\operatorname{Ln}(100)$ \\
\hline
\end{tabular}

Fuente: DEMyc, Universidad Autónoma Chapingo.

*Ln: función logaritmo.

La fórmula es la propuesta por las Naciones Unidas, que le asigna el mismo peso a salud, educación e ingreso.

Índice de desarrollo relativo al género

Mientras el idhp mide el progreso medio, el idg ajusta éste para reflejar la inequidad del desarrollo entre hombres y mujeres.

La elaboración del idg consiste en calcular para cada componente (esperanza de vida, educación e ingreso) los indicadores e índices femenino y masculino, según la fórmula (1). Para la educación se debe calcular el InEdu según la expresión (2). Los indicadores femenino y masculino para cada componente se combinan, de modo que se penalicen las diferencias en el grado de disparidad que haya entre hombres y mujeres, para que surja el índice igualmente distribuido:

índice igualmente distribuido $=\{$ proporción de población femenina (índice femenino $\left.{ }^{1-E}\right)$ ] [proporción de población masculina (índice masculino ${ }^{1-\mathrm{E}}$ )] $\}^{1 / 1-\mathrm{E}}$ 
donde E mide la aversión a la inequidad. El valor E refleja la magnitud de la penalización para la inequidad de género. Cuanto mayor sea su valor, tanto más se castiga a una sociedad por tener desigualdades.

Si $\mathrm{E}=0$, la inequidad de género no se penaliza (en este caso, el idg tendría el mismo valor que el idhp). A medida que E aumenta, se asigna una ponderación mayor al grupo menos adelantado en el factor correspondiente.

Para calcular el idg (así como el ipg) se usa el valor 2, que asigna una penalización moderada a la inequidad de género en cuanto al progreso.

Por último, se calcula el idg combinando los tres índices igualmente distribuidos en un promedio no ponderado.

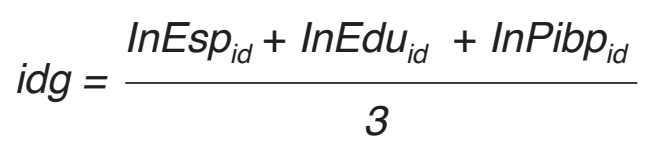

donde:

$I n E s p_{i d}$ : indicador de esperanza de vida igualmente distribuido

InEdu ${ }_{i d}$ : índice de educación igualmente distribuido

InPibp ${ }_{i d}$ : indicador del producto interno bruto per cápita igualmente distribuido.

El grado de inequidad entre hombres y mujeres se clasifica por medio de la diferencia porcentual entre el idhp y el idg en valor absoluto, es decir:

$$
d=\frac{l d h p-i d g}{i d h p} \times 100
$$

La cual proporciona la pérdida porcentual de desarrollo atribuible a la inequidad de género.

Si $d \leq 1 \%$, existe una inequidad no significativa, es decir, la diferencia de oportunidades de desarrollo entre hombres y mujeres es mínima. 
Si $1 \%<d \leq 5 \%$, hay una inequidad significativa

Si $5 \%<d$, hay una inequidad altamente significativa.

Índice de empoderamiento de género

El ipg se concentra en la participación económica, política y profesional, y difiere del idg, que se refiere a la capacidad básica y los niveles de vida.

El ipg incorpora tres clases de variables:

- En lo relativo al poder sobre los recursos económicos, basado en el ingreso proveniente del trabajo, la variable es el Pibp en dólares PPA, pero sin descontar por rendimientos decrecientes como en el idg.

- En el acceso a oportunidades profesionales y participación en la adopción de decisiones económicas, son los empleos clasificados como profesionales y técnicos y los administrativos y ejecutivos.

- En cuanto a oportunidades políticas y participación en la adopción de decisiones políticas, es la proporción de escaños parlamentarios.

Para cada variable se calcula un porcentaje equivalente igualmente distribuido (edep por sus siglas en inglés):

edep $=\left\{\begin{array}{l}\left.[\text { proporción de población femenina (índice femenino })^{1-\mathrm{E}}\right]+{ }^{1 / 1} 1-\mathrm{E} \\ \left.[\text { proporción de población masculina (índice masculino })^{1-\mathrm{E}}\right]\end{array}\right\}^{1}$

Para la participación en cuestiones políticas y económicas y en la toma de decisiones, el edep se indexa dividiéndolo por 50; la justificación de la indexación se debe a que una sociedad ideal sería en la que ambos sexos tuvieran las mismas oportunidades, en cuyo caso las variables del ipg serían iguales a 50 por ciento.

Por último, se calcula el ipg combinando los tres índices igualmente distribuidos en un promedio no ponderado. 


$$
i p g=\frac{i r p-i c_{\text {aept }}+i i i d}{3}
$$

donde los indicadores son:

iiid: ingreso igualmente distribuido

$i c_{\text {aept }}$ : combinación de puestos administrativos, ejecutivos y profesionales y técnicos

irp: representación parlamentaria.

\section{Resultados}

La región norte de México posee una extensión territorial de 518 $306 \mathrm{~km}^{2}$; una población de 7245761 habitantes; $3660511 \mathrm{mu}-$ jeres y 3585250 hombres (INEGI 2005); la cantidad de municipios permaneció constante en los años del estudio, un total de 144.

Índice de desarrollo relativo al género, 1995-2005

En 1995, el PNUD incorporó al idhp el idg, el cual penaliza la desigualdad entre hombres y mujeres. Se ha visto que en todos los países disminuyen los valores del idg en comparación con el idhp, eso significa que existe algún grado de desigualdad, ya que en el caso de producirse la equiparación total entre hombres y mujeres los valores de ambos serían idénticos.

En el idg, la brecha de género más grande es la del producto interno bruto (PIB), seguida por la de salud y por último la educación, que podría cerrarse muy pronto (Social Watch 2008). El orden coincide con el grado de inequidad existente en la distribución de estas variables en la población.

En 1995, México al igual que los estados de la región norte, registraron un idg menor que el idhp, lo cual señala una tendencia hacia la inequidad, en general contra las mujeres. En el primer quinquenio, las tres entidades de la región y el país presentaron inequidad significativa de género (la diferencia porcentual entre sus respecti- 
vos idhp e idg fue mayor a 1 por ciento), aunque las oportunidades para las mujeres en Coahuila y Chihuahua fueron superiores a las del promedio nacional. La menor pérdida en el idhp por inequidad de género de ambas entidades podría atribuirse a su ubicación geográfica, en la frontera de Estados Unidos, donde se genera un mayor desarrollo económico promovido por el intercambio comercial, lo que marginalmente permite a todos(as), y en particular a las mujeres, ser más "partícipes y beneficiarias" del desarrollo que las de otros estados del país.

\section{Figura 2}

Pérdida en el idhp por inequidad de género, región norte 1995-2005

\begin{tabular}{|c|l|c|c|c|c|}
\hline \multirow{4}{*}{1995} & & idhp & idg & $\%$ & Rango nacional \\
\cline { 2 - 6 } & Nacional & 0.770 & 0.751 & 2.44 & \\
\cline { 2 - 6 } & Chihuahua & 0.792 & 0.775 & 2.04 & 6 \\
\cline { 2 - 6 } & Coahuila & 0.806 & 0.788 & 2.19 & 7 \\
\cline { 2 - 6 } & Durango & 0.783 & 0.762 & 2.72 & 15 \\
\hline \multirow{4}{*}{2000} & Nacional & 0.792 & 0.782 & 1.27 & \\
\cline { 2 - 6 } & Chihuahua & 0.815 & 0.806 & 1.10 & 3 \\
\cline { 2 - 6 } & Coahuila & 0.826 & 0.815 & 1.24 & 11 \\
\cline { 2 - 6 } & Durango & 0.801 & 0.791 & 1.25 & 16 \\
\hline \multirow{3}{*}{2005} & Nacional & 0.799 & 0.793 & 0.728 & \\
\cline { 2 - 6 } & Chihuahua & 0.834 & 0.828 & 0.778 & 20 \\
\cline { 2 - 6 } & Coahuila & 0.817 & 0.809 & 0.961 & 29 \\
\cline { 2 - 6 } & Durango & 0.811 & 0.803 & 0.997 & 30 \\
\hline
\end{tabular}

Fuente: cálculos propios, con datos del INEGI (1995, 2000 y 2005) y Banco de Información Económica, BIE (2008).

El ingreso no es el único factor que explica la equidad ni la falta de ella, tampoco un ingreso alto es garantía de equidad de género, ni uno bajo es condición suficiente que justifique la brecha entre mujeres y hombres (Social Watch 2008). Por lo general, las mujeres han sido discriminadas en cuanto a su participación en el mercado laboral, con menor salario y acceso desigual a la toma de decisiones en las esferas económicas y políticas, además de realizar un trabajo "invisible" y no valorado, referido como doméstico (Ibarrarán y Robles 2004, 6). 
Las inequidades también están en el tipo de empleo, y es ahí donde se llega a una subordinación laboral, sobre todo de las mujeres. Según el INEGI (2005), las personas de los estados de la región norte se ocupan en actividades de los sectores secundario y terciario. Por ejemplo, en Chihuahua 32.8 por ciento de mujeres trabajan en el secundario, el más alto a escala nacional, así como 41.2 de los hombres de Coahuila, porcentaje que también es el más alto del país. Mientras que más de la mitad de hombres y mujeres se emplean en el terciario (INEGI-Instituto Nacional de las Mujeres, INMUJERES 2005).

Uno de los ejemplos más claros de la región es la participación de las mujeres en el sector agroexportador y maquilador. Este tipo de trabajo constituye la ventaja para los empresarios de contar con mano de obra barata, pero sobre todo que es muy flexible " $[\ldots]$ esta flexibilidad hace que se cuente con una mano de obra capaz de pasar de un producto a otro y de una fase del proceso productivo a otra, sin que sea reconocida su calificación o especialización en el trabajo y sin contar con ningún tipo de protección laboral $[\ldots]$ haciendo de ellas una población cautiva, y permite su contratación en condiciones de extrema precariedad" (Lara Flores 1996, 81).

Este tipo de trabajo también se caracteriza por ser a domicilio, lo que le permite a las empresas liberarse de gastos. En este sentido, paga una mínima parte a las mujeres de lo obtenido, y prolonga al doble las jornadas laborales. En este empleo flexibilizado no hay lugar para programas de previsión social, pensiones, jubilaciones o cualquier otra forma de visión de futuro con independencia económica. Se finca, asimismo, la reducción de servicios públicos esenciales para la vida, como los de salud, alimentación, cuidado infantil, agua y saneamiento en zonas rurales y el traslado de la atención de estas necesidades a manos de las mujeres.

Sin embargo, en la región estudiada, en el año 2000 se registró una inequidad de género menor a la nacional; Chihuahua tuvo menos desigualdades. En Durango se redujo más la diferencia porcentual en el primer quinquenio; disminuyó 1.47 por ciento (de 2.72 pasó a 1.25); no obstante los avances no fueron suficientes para mejorar su clasificación y alcanzar una inequidad no significativa de género, es decir, las mujeres de Chihuahua, Coahuila y Durango participaron más activamente en el sector económico y 
por lo tanto obtuvieron retribuciones monetarias, aunque el tipo de empleo tenga desventajas, como incrementar su carga de trabajo. No obstante, más allá de un nivel moderado, ellas siguen viviendo de forma diferenciada, desfavorable e injusta por razones de género.

La inequidad de género en la región norte se torna más diversa cuando el análisis se hace a escala municipal, en la figura 3 se da cuenta de ello. Esta inequidad algunas veces resulta favorable a las mujeres, como sucedió en Matachi en 1995, donde ellas vivieron en promedio 8.72 años más que los hombres, su índice de educación fue mayor en 1.2 por ciento al de ellos, pero con un Pibp que representa 19.5 del generado por hombres, es decir, en esperanza de vida y educación ellas se encontraron en mejor condición, pero en generación de ingresos la diferencia es muy grande y al promediar los tres índices, con el mismo peso, la ventaja fue para las mujeres.

Otra característica de los municipios con menor inequidad es la diferencia mínima del Pibp entre géneros, tal es el caso de la ciudad

\section{Figura 3}

Municipios con menor pérdida de idhp por inequidad de género

\begin{tabular}{|c|c|c|c|c|c|c|c|c|}
\hline & \multicolumn{2}{|c|}{$\begin{array}{l}>50000 \\
\text { habitantes }\end{array}$} & \multirow[b]{2}{*}{$\%$} & & \multicolumn{2}{|c|}{$\begin{array}{l}<50000 \\
\text { habitantes }\end{array}$} & \multirow[b]{2}{*}{$\%$} \\
\hline \multirow{5}{*}{1995} & & idhp & idg & & & idhp & idg & \\
\hline & Acuña, Coah. & 0.788 & 0.776 & 1.45 & A. Serdán, Chih. & 0.786 & 0.785 & 0.13 \\
\hline & Ciudad Juárez, Chih. & 0.795 & 0.782 & 1.67 & Matachi, Chih. & 0.753 & 0.760 & 0.95 \\
\hline & Chihuahua, Chih. & 0.817 & 0.803 & 1.70 & Villa Unión, Coah. & 0.779 & 0.769 & 1.30 \\
\hline & Piedras Negras, Coah. & 0.807 & 0.792 & 1.83 & Sta. Isabel, Chih. & 0.800 & 0.789 & 1.32 \\
\hline \multirow{4}{*}{2000} & Chihuahua, Chih. & 0.839 & 0.831 & 0.89 & El Tule, Chih. & 0.777 & 0.777 & 0.01 \\
\hline & Acuña, Coah. & 0.813 & 0.806 & 0.90 & Rosario, Chih. & 0.779 & 0.779 & 0.01 \\
\hline & Durango, Dgo. & 0.816 & 0.808 & 1.01 & Matachi, Chih & 0.794 & 0.793 & 0.08 \\
\hline & San Pedro, Coah. & 0.792 & 0.784 & 1.09 & C. de Comonfort, Dgo. & 0.718 & 0.717 & 0.14 \\
\hline \multirow{4}{*}{2005} & Acuña, Coah. & 0.832 & 0.828 & 0.53 & Dr. Belisario D., Chih. & 0.800 & 0.800 & 0.03 \\
\hline & Torreón, Coah. & 0.839 & 0.832 & 0.72 & El Tule, Chih. & 0.780 & 0.779 & 0.05 \\
\hline & Piedras Negras, Coah. & 0.828 & 0.822 & 0.76 & V. de Zaragoza, Chih. & 0.793 & 0.792 & 0.11 \\
\hline & Saltillo, Coah. & 0.843 & 0.836 & 0.78 & Rosario, Chih. & 0.788 & 0.787 & 0.14 \\
\hline
\end{tabular}

Fuente: cálculos propios, con datos del INEGI (1990, 1995, 2000 y 2005); BIE (2008). 
de Chihuahua, que en el año 2000 había alcanzado una inequidad no significativa. El Pibp generado por mujeres representó 43 por ciento del de hombres (uno de los ingresos más altos para mujeres en la región), en el acceso a la educación fue relativamente la misma para ambos y en esperanza de vida ellas superaron con 6.17 años a los hombres.

En 1995, 1 por ciento (7 289 habitantes) de la población en dos municipios de la región alcanzó una inequidad de género no significativa, cinco años después aumentó a 13.89 por ciento (944966 habitantes) en 29 municipios. En 2005, 67.7 por ciento (4 906110 habitantes) ditribuidos en 50 municipios presentaron poca inequidad. Aunque éstos no mostraron grandes disparidades entre hombres y mujeres en el desarrollo de sus capacidades, no implica que gocen de mejor DH, lo cual se observa en los índices obtenidos.

El problema presente en los municipios con mayor inequidad de género fue la brecha entre el Pibp generado por hombres y mujeres, que se marcó en aquéllos con poca población, como en Riva Palacio, donde en 2005 aumentó la diferencia del Pibp y por ende su inequidad de género (véase figura 4).

En los municipios con más de 50 mil habitantes, las oportunidades para el desarrollo son menos diferenciadas entre hombres y mujeres, puesto que en esos lugares es más fácil proporcionar recursos a la población, porque están comunicados por carreteras y cuentan con infraestructura, lo que abre un abanico de posibilidades. Pero, ¿qué pasa en municipios pequeños y de difícil acceso? En ellos se establece un círculo vicioso entre una aportación mínima a la economía local y el progreso de su comunidad, tanto humano como en infraestructura, lo que les dificulta verse favorecidos con programas públicos en beneficio de la salud, educación y empleo. En estos lugares se ha visto que las mujeres son las más perjudicadas, lo que se traduce en un DH menor al de los hombres.

Educación

La educación debe ser un derecho para todos y todas, para obtener los conocimientos mínimos necesarios para acceder a un trabajo y 
percibir un ingreso que mejore el nivel de vida. Según Social Watch (2008), la educación es el único componente del índice en el cual muchos países han alcanzado una paridad. Sin embargo, también es importante considerar qué calidad de educación se imparte y la segregación de ésta en las disciplinas y niveles educativos (sobre todo en los más altos).

\section{Figura 4}

Municipios con mayor pérdida de idhp por inequidad de género

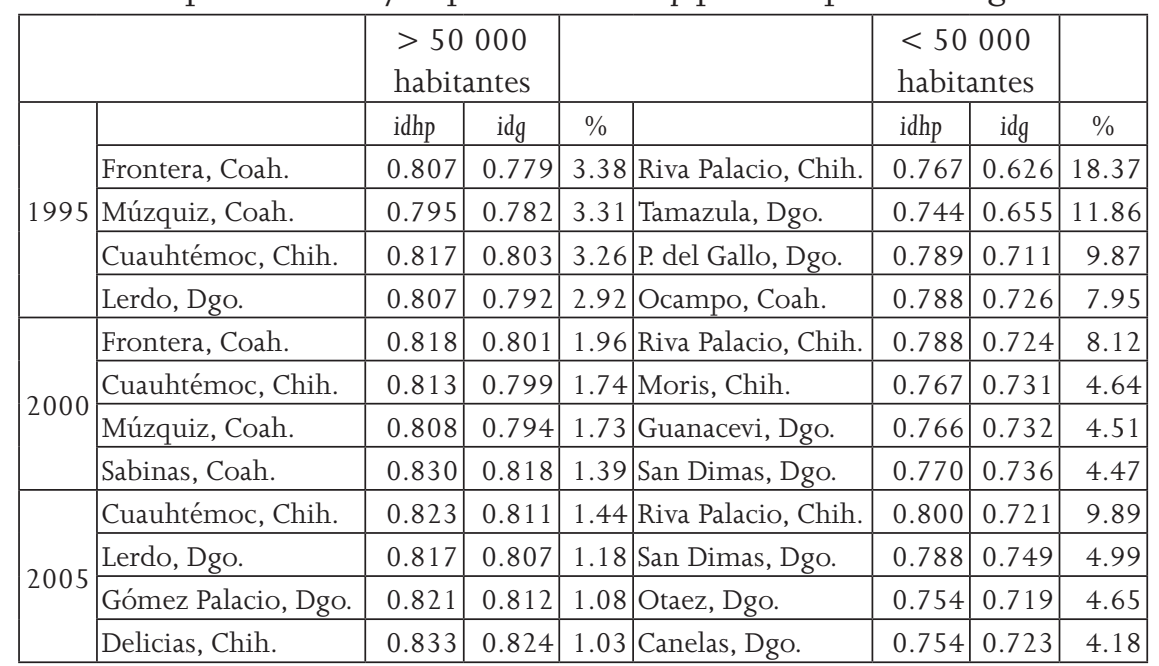

Fuente: cálculos propios, con datos del INEGi (1990, 1995, 2000 y 2005); BIE (2008).

Aun cuando en México no hay diferencia por género en la educación, siguen existiendo inequidades para que las mujeres ingresen a estudios como el superior y posgrado. Asimismo, hay estereotipos a la hora de elegir una profesión, pues las mujeres siguen prefiriendo las feminizadas como sociología, psicología, trabajo social, docencia y enfermería, carreras bien aceptadas socialmente para ellas, ocupaciones que les permiten no estar muy lejos del hogar (Millán 2001, 307).

La educación en general es indispensable en el desarrollo social y personal de los habitantes de un país, sin embargo, la de las mujeres juega un papel importante en el bienestar de los otros, es decir, que 
ellas cuenten con un grado de escolaridad mayor está relacionado directamente con el bienestar, salud, nutrición y educación de los miembros de su hogar o familia. Por ejemplo, la eliminación del analfabetismo entre las mujeres no puede separarse de la erradicación de la pobreza, así como su educación y empleo se consideran factores importantes para reducir las tasas de fertilidad (Kabeer 2006, 30).

El avance productivo también está relacionado con la educación. Sin embargo, el logro educativo por sí mismo no significa la obtención automática de mejores puestos de trabajo o más poder en el campo personal, social o político de las mujeres. Aunque su presencia en el ámbito educativo las ha conducido a alcanzar un mayor poder de acción y autonomía, con lo que han mejorado su posición.

\section{Figura 5}

Inequidad en la educación, región norte

\begin{tabular}{|c|c|c|c|c|}
\hline & Hombres & Mujeres & Diferencia \\
\hline & & InEdu $\mathrm{m}$ & InEdu $\mathrm{f}$ & $(\%)$ \\
\hline \multirow[t]{6}{*}{1995} & Nacional & 0.895 & 0.869 & 2.5 \\
\hline & Coahuila & 0.935 & 0.935 & 0.0 \\
\hline & Chihuahua & 0.922 & 0.924 & 0.2 \\
\hline & Durango & 0.913 & 0.915 & 0.2 \\
\hline & Guadalupe, Chih. & 0.904 & 0.904 & 0.0 \\
\hline & Guachochi, Chih. & 0.670 & 0.592 & 7.8 \\
\hline \multirow[t]{6}{*}{2000} & Nacional & 0.905 & 0.883 & 2.2 \\
\hline & Coahuila & 0.943 & 0.943 & 0.0 \\
\hline & Chihuahua & 0.934 & 0.937 & 0.3 \\
\hline & Durango & 0.923 & 0.927 & 0.4 \\
\hline & Riva Palacio, Chih. & 0.957 & 0.957 & 0.0 \\
\hline & Mezquital, Dgo. & 0.736 & 0.586 & 15.0 \\
\hline \multirow[t]{6}{*}{2005} & Nacional & 0.911 & 0.894 & 1.68 \\
\hline & Coahuila & 0.945 & 0.947 & 0.2 \\
\hline & Chihuahua & 0.934 & 0.936 & 0.2 \\
\hline & Durango & 0.928 & 0.931 & 0.4 \\
\hline & Monclova, Coah. & 0.961 & 0.961 & 0.0 \\
\hline & Mezquital, Dgo. & 0.754 & 0.626 & 12.8 \\
\hline
\end{tabular}

Fuente: cálculos propios, con datos del INEGI (1990, 1995, 2000 y2005); BIE (2008). 
En el país, las oportunidades en educación favorecieron a los hombres, pero a escala regional las mujeres tuvieron una ligera ventaja; Durango obtuvo la diferencia máxima. Aunque en la mayor parte de los municipios la educación se inclinó a favor del sexo femenino, hubo otros donde sucedió lo contrario, con índices bajos, como en Mezquital y Guachochi, donde el avance de la educación está limitado por situarse en zonas alejadas, montañosas o de difícil acceso. En las comunidades alejadas las inequidades son más grandes, porque no cuentan con los recursos e infraestructura necesarios para educar a niños y niñas y, por ende, la deficiencia en el servicio ofrecido es mayor.

Esperanza de vida

La esperanza de vida es una medida resumen sobre la mortalidad, que expresa el promedio de años que se espera viva una persona en situaciones de mortalidad del periodo en que se calcula, y constituye un indicador para caracterizar las condiciones de vida, salud, educación y otros elementos sociales de un país. Estas características han hecho que la esperanza de vida sea uno de los indicadores fundamentales, seleccionado por las Naciones Unidas para medir el desarrollo humano.

Este fenómeno tiene determinantes biológicos y socioeconómicos, se relaciona en forma directa con el lugar de residencia, la escolaridad de la población y prácticas sociales y culturales. La mayor esperanza de vida en las mujeres tiene connotaciones de sexo y género. En lo sexual, la propia biología e incluso la genética determinan la longevidad de mujeres y hombres, sin embargo son muy importantes los factores ambientales, modificables, determinados en gran parte por las relaciones de género. Por ejemplo, por lo general los hombres se emplean en trabajos de mayor riesgo y donde se necesita fuerza física, por lo que su cuerpo se desgasta más, aunado a ello los estereotipos de machismo han provocado que se enfrenten a situaciones más violentas y riesgosas en su vida diaria, como riñas, accidentes automovilísticos o de otro tipo, cuya consecuencia es una esperanza de vida menor a la de las mujeres. 
A escala nacional y regional, la esperanza de vida femenina en la mayoría de los municipios fue superior a la masculina; Coahuila mostró menor inequidad de género en salud, y Chihuahua el mayor.

Según estadísticas de INEGI-INMUJERES (2005), en el tema de sobremortalidad masculina, para 2002, en Coahuila morían 121 hombres por 100 mujeres, sin embargo la situación se agravó en las edades de 15 a 29 años, cuando hubo 277.8 defunciones masculinas por cada 100 femeninas. En Chihuahua, el índice de sobremortalidad fue de 138.1 y en Durango de 140.3, y aumentó en la población de 15 a 29 años a 283.7 y 260.6 respectivamente.

\section{Figura 6}

Inequidad en esperanza de vida, región norte

\begin{tabular}{|c|l|c|c|c|}
\hline \multicolumn{1}{|c|}{1995} & Hombres & Mujeres & Diferencia(en años) \\
\cline { 2 - 5 } & Nacional & 70.1 & 75.0 & 4.9 \\
\cline { 2 - 5 } & Coahuila & 71.6 & 75.1 & 3.4 \\
\cline { 2 - 5 } & Durango & 72.1 & 77.2 & 5.1 \\
\cline { 2 - 5 } & Chihuahua & 68.7 & 74.3 & 5.5 \\
\cline { 2 - 5 } & Nava, Coah. & 73.2 & 73.2 & 0.0 \\
\cline { 2 - 5 } & Uruachi, Chih. & 63.5 & 79.4 & 15.8 \\
\hline \multirow{3}{*}{2000} & Nacional & 71.5 & 76.2 & 4.7 \\
\cline { 2 - 5 } & Coahuila & 72.3 & 76.0 & 3.6 \\
\cline { 2 - 5 } & Durango & 73.2 & 77.8 & 4.5 \\
\cline { 2 - 5 } & Chihuahua & 69.2 & 74.8 & 5.6 \\
\cline { 2 - 5 } & Nava, Coah. & 76.0 & 76.2 & 0.2 \\
\cline { 2 - 5 } & Batopilas, Chih. & 55.2 & 71.5 & 16.2 \\
\cline { 2 - 5 } & Cacional & 72.3 & 76.9 & 4.5 \\
\cline { 2 - 5 } & Doahuila & 73.0 & 76.3 & 3.3 \\
\cline { 2 - 5 } & Chihuahua & 73.6 & 78.1 & 4.4 \\
\cline { 2 - 5 } & Castaños, Coah. & 75.7 & 75.3 & 5.6 \\
\cline { 2 - 5 } & Guadalupe y Calvo, Chih. & 59.3 & 77.3 & 0.19 \\
\hline
\end{tabular}

Fuente: cálculos propios, con datos del INEGI (1990, 1995, 2000 y 2005); BIE (2008).

En la figura 6 se aprecia que los hombres en Chihuahua registraron una esperanza de vida muy baja, quizá por el grado de delin- 
cuencia en el estado, además de los riesgos que corren en el trabajo y accidentes al salir de casa. Fue la sexta entidad más violenta del país en el año 2000; de 588 homicidios en ese año, 530 fueron de hombres, según las estadísticas vitales del inEGi (2000). Por lo general, los municipios de la región norte con mayor diferencia en esperanza de vida tienen menos de 50 mil habitantes (Uruachi 8587 y Batopilas 12 595), hecho que puede estar influido por las variaciones inestables en poblaciones pequeñas.

Producto interno bruto per cápita

La inserción de las mujeres en el mercado de trabajo y la obtención de recursos económicos es de gran importancia para aumentar el grado de autonomía y bienestar, propios y de sus familias (Abramo y Valenzuela 2006, 31).

La discriminación económica y la segregación ocupacional están relacionadas con las desigualdades de género en el mercado laboral, y como consecuencia en la generación de ingresos. La primera está vinculada con las diferencias en las remuneraciones atribuidas a aspectos como sexo, raza, etnia y edad. La segunda se refiere a la concentración de las mujeres en cierto tipo de ocupaciones "feminizadas" o, dicho de otro modo, su exclusión de ciertas actividades productivas. La discriminación y segregación laboral descansan en construcciones culturales e institucionales de los mercados de trabajo, y están basadas en el sistema de género de las sociedades. Asimismo, explica las diferencias en las remuneraciones promedio entre hombres y mujeres (PNUD 2008, 226).

En el sostenimiento y reproducción de las desigualdades entre hombres y mujeres en el mundo del trabajo inciden varios factores, derivados de la división sexual del mismo y del orden de género (que incluye también todos los otros ámbitos de la vida social). A la mujer se le asigna la función básica y primordial del cuidado con el mundo privado y la esfera doméstica, a la cual se le atribuye un valor social inferior al mundo "público" (y se desconoce por completo su valor económico). Eso, para ellas no sólo significa una limitación de tiempo y recursos para invertir en su formación y trabajo remunerado, sino que también está relacionado a una subva- 
loración (económica y social) del trabajo y de su rol en la sociedad (Abramo y Valenzuela 2006, 32). Las valoraciones diferenciadas sociales y económicas del trabajo, tanto remunerado como no pagado, promueven un reparto de cargas, tiempos, rentas y derechos que resultan discriminatorios para muchas (PNUD 2008, 226).

La brecha en la percepción de ingresos es la más alta en los índices de desarrollo humano, sin embargo, para su cálculo no se ha considerado el trabajo reproductivo, que por lo general es realizado por las mujeres, no es valorado ni económica ni socialmente, y siempre ha sido excluido del análisis económico. Hay una tendencia a considerarlo como algo "natural" de las obligaciones de las mujeres, no un trabajo en sí, porque no es remunerado. Al basar la economía en el hombre, el análisis económico ha tenido desviaciones y no ha sabido apreciar el rasgo distintivo que tienen los patrones de trabajo de las mujeres. Esto ha hecho que se tenga un panorama de iceberg de la economía, pues sólo se ve la punta de todo lo que constituye el trabajo productivo (Kabeer 2006, 45). El rezago de éste en las mujeres es muy marcado, y para los cálculos del Pib per cápita en el idhp e idg no se toma en cuenta su trabajo reproductivo.

En la región de estudio, la brecha, en términos de capacidad de generar ingresos tanto a escala regional como nacional, favoreció a los hombres pero con una tendencia positiva para las mujeres. En este ámbito es donde se ve más la diferencia inequitativa entre lo que ambos perciben o de las oportunidades de ellas para acceder a un empleo bien remunerado y desarrollar sus capacidades. De los municipios con menos de 50 mil habitantes, resalta Riva Palacio (10 020), cuya diferencia del Pibp entre hombres y mujeres fue una de las más críticas en 1995 y 2005; tuvo los porcentajes femeninos más bajos, 2.33 y 5.91 respectivamente.

Según Social Watch (2008), la brecha en la actividad económica de hombres y mujeres parece ir en aumento en el mundo, ya que más de la mitad de los países experimentan retroceso. Esta regresión es la mayor responsable del repliegue de los países en la evolución general del idg. Lo anterior contrasta con los resultados en la región analizada.

Un resumen de la región muestra que en educación no hay mucha desigualdad; el ingreso superior de los varones obnubila la po- 
sible ventaja que las mujeres tienen en salud, y eso determina el grado de inequidad, que es mayor en los municipios que en los estados.

\section{Figura 7}

Inequidad en el Pibp, región norte

\begin{tabular}{|c|c|c|c|c|}
\hline & & Hombres & Mujeres & $\%^{*}$ \\
\hline \multirow{6}{*}{1995} & Nacional & 19938 & 5112 & 25.6 \\
\hline & Chihuahua & 15031 & 4227 & 28.1 \\
\hline & Coahuila & 15198 & 3967 & 26.1 \\
\hline & Durango & 10296 & 2418 & 23.4 \\
\hline & Ignacio Zaragoza, Chihuahua & 11263 & 3976 & 35.3 \\
\hline & Riva Palacio, Chihuahua & 9993 & 233 & 2.3 \\
\hline \multirow{6}{*}{2000} & Nacional & 13360 & 4973 & 37.22 \\
\hline & Chihuahua & 18637 & 7331 & 39.3 \\
\hline & Durango & 10946 & 4090 & 37.3 \\
\hline & Coahuila & 17686 & 6348 & 36.0 \\
\hline & Ignacio Zaragoza, Chihuahua & 12116 & 6794 & 56.0 \\
\hline & Riva Palacio, Chihuahua & 11999 & 915 & 7.6 \\
\hline \multirow{6}{*}{2005} & Nacional & 12307 & 5862 & 47.6 \\
\hline & Coahuila & 18129 & 8082 & 44.6 \\
\hline & Chihuahua & 18006 & 7503 & 41.7 \\
\hline & Durango & 11990 & 4923 & 41.1 \\
\hline & Ignacio Zaragoza, Chihuahua & 11634 & 8028 & 69.0 \\
\hline & Riva Palacio, Chihuahua & 13279 & 785 & 5.9 \\
\hline
\end{tabular}

* Por ejemplo, en Durango el Pibp femenino representa 23.4 por ciento del Pibpm. Fuente: cálculos propios, con datos del INEGI (1990, 1995, 2000 y 2005); BIE (2008).

Inequidad de la inequidad

Al grado de inequidad relacionado de manera inversa con el de desarrollo se le puede llamar inequidad de la inequidad, y muestra las interacciones negativas con un DH bajo por ser donde se localizan las mayores inequidades de género, es decir, los lugares donde hay menos oportunidades de crecer inducen una inequidad entre hombres y mujeres que, a su vez, dificulta el desarrollo de las personas conformando un círculo vicioso que lo mantiene bajo (véase figura 8). 
Figura 8

Inequidad de la inequidad en la región norte

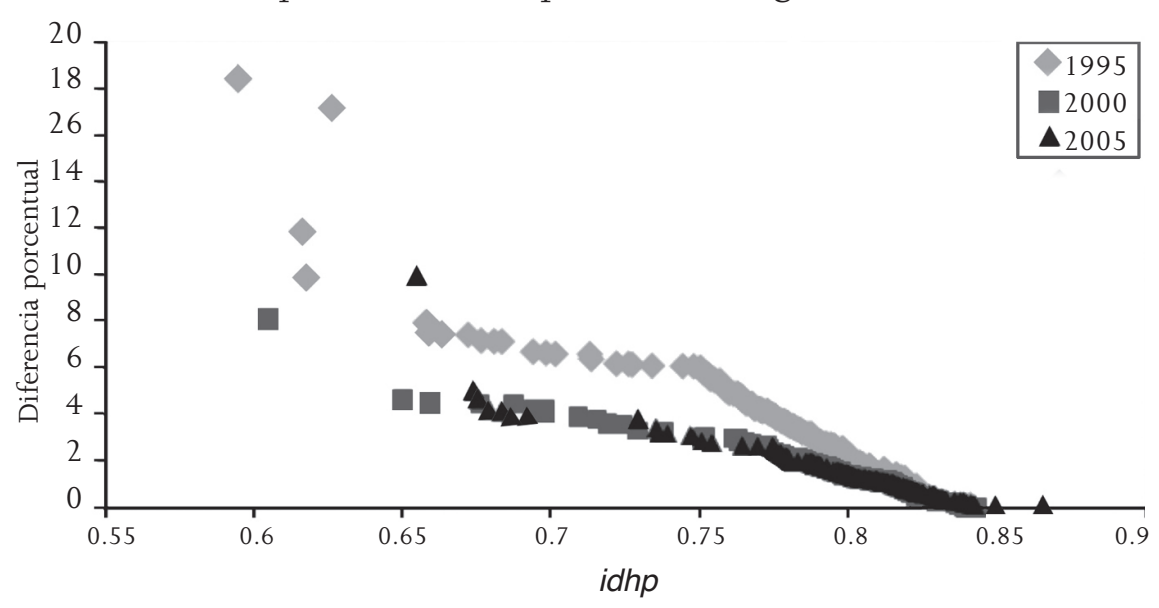

Fuente: cálculos propios, con datos del INEGI (1990, 1995, 2000 y 2005); BIE (2008).

Lo anterior permite inferir que si un municipio mejora su condición de DH a través del tiempo, también lo hará su equidad y viceversa, transformando un círculo vicioso en uno virtuoso. Asimismo, exhibe que la carencia de opciones determina en alguna medida una conducta inequitativa.

A partir de un idhp=0.75 la caída en inequidad se acelera, la cual puede ser un indicativo del grado de desarrollo necesario que manifiesta aversión a la inequidad y trata de corregirla. Para saber más o menos cuánto disminuyó la inequidad de género al ir aumentando el valor del idhp, se obtuvo una ecuación que ajustó el comportamiento de los datos para 1995, 2000 y 2005.

De la ecuación correspondiente a 2005, se dice que para un desarrollo nulo la inequidad máxima pudo haber sido de 23.6 por ciento y por cada 0.1 que aumentó el idhp, la inequidad disminuyó en 2.7 por ciento.

Puesto que la inequidad de género depende en forma directa del DH, esta dependencia brinda una idea de cómo contrarrestarla, es ahí donde las autoridades y la sociedad en general deben esforzarse por 
lograr un mayor desarrollo con equidad, en especial en los lugares con índices de desarrollo bajos con una inequidad yuxtapuesta.

\section{Figura 9}

Relación de la inequidad de género con el desarrollo

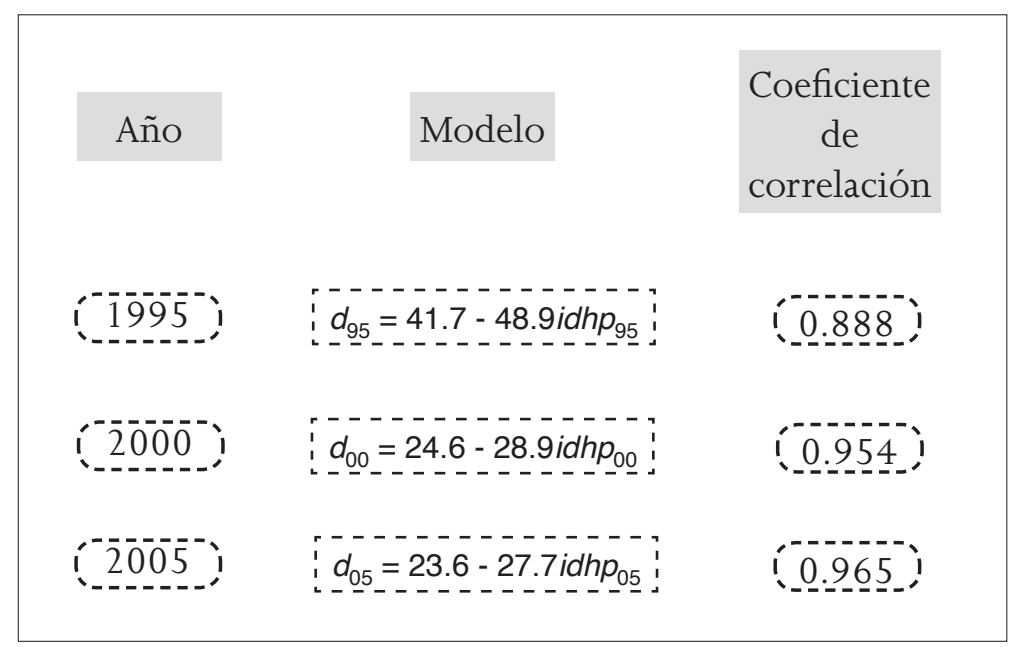

Fuente: cálculos propios, con datos del INEGI (1990, 1995, 2000 y 2005); BIE (2008).

Índice de empoderamiento de género, 2000

El ipg pretende captar la desigualdad de género en esferas clave de la participación económica, política y de la adopción de decisiones. Así, se centra más en las oportunidades que se ofrecen a las mujeres que en sus capacidades. En cierta forma, puede decirse que el ipg resulta más un indicador de empoderamiento que de bienestar.

En la Conferencia Mundial de las Naciones Unidas sobre la Mujer (Beijing 1995), el empoderamiento se planteó como condición necesaria para construir un mundo mejor para ellas (Townsend 2002, 38). Desde la perspectiva de género, Batliwala (1993, citada por Sen, 1996) considera que el empoderamiento posee dos elementos centrales de control: sobre los recursos (físicos, humanos, intelectuales, financieros y el propio ser) y sobre la ideología (creencias, 
valores y actitudes). Si el poder significa control, el empoderamiento, por tanto, es el proceso de ganar control.

Para medir el empoderamiento de las mujeres, los reportes de DH han tomado en cuenta su participación en la vida política y profesional, su autonomía en la toma de decisiones y en la distribución del trabajo entre hombres y mujeres (López 2006, 4). El PNUD agregó a sus índices el de empoderamiento de género (ipg), con base en cuatro indicadores de porcentaje de las mujeres: en cargos técnicos; en cargos de dirección y gobierno; en el parlamento y en cargos ministeriales.

En la región estudiada, el mayor ipg lo tuvo Chihuahua, similar al de Singapur (0.592), el segundo fue para Coahuila, comparable con la República Checa (0.560), Durango estuvo a la zaga, equiparable con el de Uruguay (0.519). El ipg nacional se comparó con el de Filipinas (0.523) (PNUD 2002).

\section{Figura 10}

Empoderamiento de género, 2000

\begin{tabular}{|l|c|c|c|}
\hline Regional & ipg & Nacional & ipg \\
\hline Chihuahua & 0.594 & País & 0.523 \\
\hline Coahuila & 0.559 & D. F. & 0.695 \\
\hline Durango & 0.518 & Chiapas & 0.405 \\
\hline \multicolumn{3}{|c|}{ Escala municipal } \\
\hline Hidalgo del Parral, Chih. & 0.726 & Benito Juárez, D. F. & 0.795 \\
\hline Maguarichi, Chih. & 0.025 & San Andrés Duraznal, Chiapas & 0.000 \\
\hline
\end{tabular}

Fuente: cálculos propios, con datos del INEGI (2000); BIE (2008).

Como se observa, la inequidad más grave que sufren las mujeres es la relacionada con el empoderamiento, puesto que los valores de ipg nacionales y regionales fueron muy bajos. El municipio con mayor empoderamiento femenino fue Hidalgo del Parral, de 100 800 habitantes, pero a pesar de encontrarse entre los 15 con mejor ipg del país, el empoderamiento masculino siguió siendo superior, pues una sociedad justa sería cuando hombres y mujeres estuvieran en condiciones equivalentes. 


\section{Figura 11}

El Pibp en el ipg, región norte

\begin{tabular}{|l|c|c|c|}
\hline & Hombres & Mujeres & Diferencia (\%) \\
\hline Nacional & 13360 & 4973 & 37.22 \\
\hline Chihuahua & 18637 & 7331 & 39.33 \\
\hline Durango & 10946 & 4090 & 37.36 \\
\hline Coahuila & 17686 & 6384 & 36.09 \\
\hline Ignacio Zaragoza, Chih. & 12116 & 6794 & 56.07 \\
\hline Riva Palacio, Chih. & 11999 & 915 & 7.63 \\
\hline
\end{tabular}

Ejemplo: en Ignacio Zaragoza, el Pibpf representa 56.07\% del Pibp masculino (cifras en dólares PPA).

Fuente: cálculos propios, con datos del INEGI (2000); BIE (2008).

Figura 12

Empoderamiento en las oportunidades profesionales, región norte

\begin{tabular}{|c|c|c|c|c|c|}
\hline & \multicolumn{2}{|c|}{ Profesionales y técnicos } & & \multicolumn{2}{|c|}{$\begin{array}{c}\text { Administrativos y } \\
\text { ejecutivos }\end{array}$} \\
\hline & Hombres \% & Mujeres \% & & Hombres \% & Mujeres \% \\
\hline Nacional & 61 & 39 & Nacional & 73 & 27 \\
\hline Durango & 56 & 44 & Durango & 72 & 28 \\
\hline Coahuila & 60 & 40 & Chihuahua & 74 & 26 \\
\hline Chihuahua & 65 & 35 & Coahuila & 74 & 26 \\
\hline \multicolumn{6}{|c|}{ Escala municipal } \\
\hline San Pedro, Coah. & 52 & 48 & Matamoros, Coah. & 68 & 32 \\
\hline $\begin{array}{l}\text { Ciudad Juárez, } \\
\text { Chih. }\end{array}$ & 70 & 30 & Sabinas, Coah. & 78 & 22 \\
\hline
\end{tabular}

Fuente: cálculos propios, con datos del INEGI (2000); BIE (2008).

El empoderamiento económico de la mujer medido por el Pibp fue el más desfasado, pues el acceso a las actividades remuneradas se ve limitado por razones de género, así como por la falta de tiempo de las mujeres y las dobles o triples jornadas de trabajo a las que están sometidas. En Ignacio Zaragoza, las mujeres tuvieron mayor presencia en el mercado de trabajo, usaron sus capacidades para generar un ingreso que mejorara su estilo de vida e igualara el empoderamiento entre ambos. 
Alcanzar un mejor nivel educativo permite insertarse en empleos mejor remunerados, sin embargo, en el idg las mujeres registraron un mayor acceso a la educación, que no se refleja en las oportunidades profesionales y la participación en decisiones económicas (véase figura 12). En este aspecto, Durango fue el que registró la mayor participación femenina, hecho que contrasta con el resto de las variables. De los municipios con una población mayor a los 50 mil habitantes, San Pedro tuvo menos restricciones en contra de las mujeres, en cuanto a los empleos clasificados como profesionales y técnicos, y Matamoros con los administrativos y ejecutivos.

La participación política de las mujeres, tanto en el ejercicio de poder como en la toma de decisiones, no fue significativa en la región, ya que éstas ocuparon 19 por ciento de los 1246 asientos en el parlamento. Chihuahua tuvo más representación parlamentaria femenina, pero con un porcentaje tan bajo que permite afirmar que las mujeres siguen representadas por los hombres en la arena política (véase figura 13).

\section{Figura 13}

Representación en cuerpos legislativos, 2000

\begin{tabular}{|l|c|c|}
\hline & Hombres & Mujeres \\
\hline Nacional & 83 & 17 \\
\hline Chihuahua & 78 & 22 \\
\hline Coahuila & 82 & 18 \\
\hline Durango & 85 & 15 \\
\hline Escala municipal & 47 & 53 \\
\hline Hidalgo del Parral, Chihuahua & 91 & 9 \\
\hline San Pedro, Coahuila
\end{tabular}

Fuente: cálculos propios, con datos del INEGI (2000); BIE (2008).

En los casos extremos, como Hidalgo del Parral, los avances logrados resultaron del derecho al voto de las mujeres, que se extendió en 1953, y de la resolución en 1996 de la Cámara de Diputados para promover que los partidos políticos en sus estatutos de las candidaturas a cargos de elección popular no se excedieran de 70 por ciento para un mismo sexo. 
En San Pedro, la falta de conocimiento o decisión femenina de emplearse en puestos que por tradición han ocupado los hombres ha disminuido su participación e incrementado el poder masculino.

En la figura 14 se exhibe la relación directa entre el desarrollo y empoderamiento de género, que ocurre cuando se ofrecen medios y recursos a hombres y mujeres para desarrollar de forma integral sus capacidades, lo cual a su vez contribuye para que ambos tengan la misma posibilidad de hacer uso de ellas.

Para conocer el aumento promedio del empoderamiento de género al ir incrementando el valor del idhp, se obtuvo una ecuación que ajustó el comportamiento de los datos del año 2000, que se incluye a continuación:

$$
i p g_{00}=3.3 i d h p_{00}-2.1
$$

Esta ecuación indica que por cada 0.1 que aumentó el valor del desarrollo humano, el empoderamiento de género creció 0.33 . El coeficiente de correlación entre las variables fue de 0.77.

\section{Figura 14}

Inequidad en el empoderamiento en la región norte

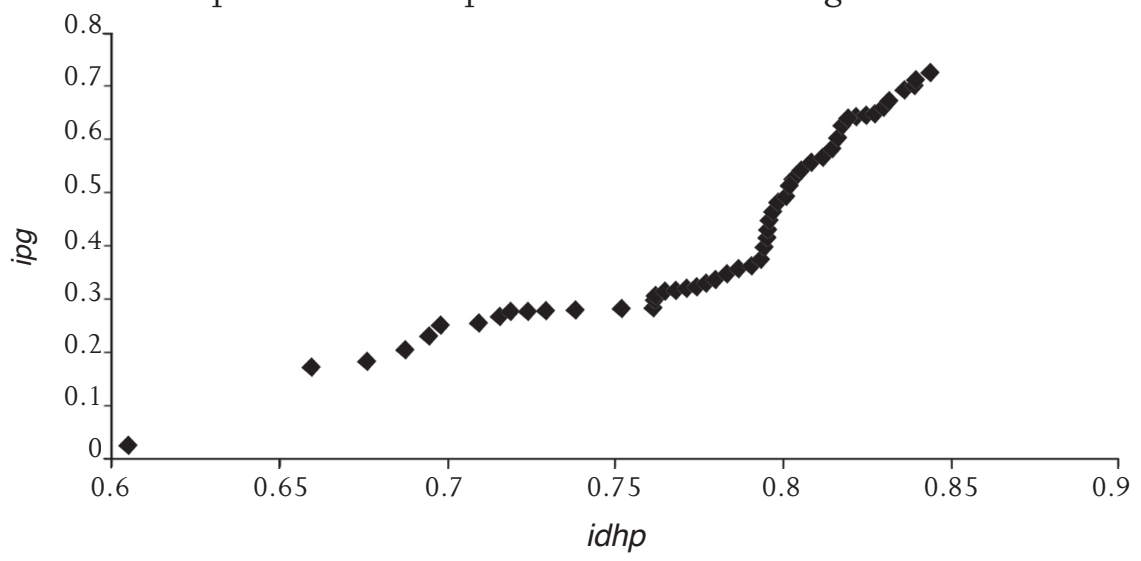

Fuente: cálculos propios, con datos del INEGI (2000); BIE (2008). 


\section{Discusión}

A pesar de las diferencias que muestran los estados de la región norte, hay elementos comunes manifiestos en su tratamiento al problema de género. De modo coincidente, los tres están alcanzando el desarrollo alto y simultáneamente una inequidad no significativa y grandes opciones de desarrollo. En 1995 tenían una posición mejor con respecto al resto de las entidades, el lugar alcanzado en 2005 fue menor, derivado del estancamiento o crecimiento lento después de 1995, así como de los problemas sociales y de violencia. En 1995, Coahuila, Chihuahua y Durango tenían las posiciones 7 , 6 y 15 respectivamente, diez años después la 20, 29 y 30 a escala nacional, pérdidas que exhiben el rezago de la zona respecto a otras del país.

\section{Desarrollo relativo al género}

Con respecto a la educación se puede decir que las oportunidades han sido similares para hombres y mujeres. Al igual que en el resto de México y la mayoría de los países, las brechas de género en cuanto a la educación se están cerrando, a tal grado que son casi imperceptibles y en ocasiones las mujeres están en ventaja con respecto a los hombres, aun cuando en las zonas rurales las diferencias son mayores. Cabe señalar que no se está calificando la calidad de la educación, sino que todos y todas puedan acceder a ella, aunque sea mala, como en muchos lugares de México.

La longevidad de las mujeres es mayor que la de los hombres, y la de Coahuila está significativamente por debajo del promedio nacional. En la esperanza de vida ellas tienen más ventaja sobre los varones, y al promediarla con la educación e ingresos equilibra las mediciones y disminuye las inequidades. Que las mujeres vivan más no quiere decir que lo hagan de la mejor manera y con calidad. Cuando se analizan los factores de riesgo durante la vejez como la falta de salud, los recursos económicos, el aislamiento o situación de dependencia, se pone de manifiesto que ellas son las que en mayor medida viven solas, son viudas o solteras, reciben pensiones inferiores a las de los varones, además sufren más por la enfermedad 
y ellas se desempeñan, hasta edades avanzadas, como cuidadoras de nietos y nietas, así como de personas en situación de dependencia.

En cuanto a la producción de recursos económicos, las brechas de género son muy grandes, de hecho este es el índice de mayor rezago y más significativo. Desde siempre, la inequidad se ha visualizado más en las labores productivas. De acuerdo con la división sexual del trabajo, a lo largo del tiempo las mujeres han sido recluidas al mundo privado-doméstico y los hombres se han desempeñado en el público; mientras que ellos laboran y obtienen remuneración económica, el trabajo reproductivo realizado por las mujeres no es valorado. Con el idg se calculó el ingreso per cápita de ambos, sin embargo, no se incorpora el cálculo del trabajo de reproducción, que por lo general realizan ellas, por lo tanto el indicador es incompleto.

La generación de recursos económicos para las mujeres ha sido de gran importancia, les ha permitido ganar terreno en la toma de decisiones tanto dentro de sus hogares como en la política, otras actividades sociales, sobre su propio cuerpo y tener mayor autonomía. No obstante, es necesario analizar y conocer las condiciones en las que las mujeres ingresan al mundo laboral remunerado, pues es común que realicen tareas subordinadas y poco remuneradas o reconocidas socialmente, son trabajos más flexibles y no cuentan con servicios de salud, prestaciones y pensiones.

Empoderamiento de género, 2000

Aunque se ha tratado de alcanzar un desarrollo análogo entre hombres y mujeres en la región norte, se reflejó una desigualdad de poder favorable para ellos. La participación activa de las mujeres tuvo mayor presencia en las oportunidades profesionales, sin embargo, no se ha logrado una equivalencia con respecto a los hombres. Las mujeres han tenido mayor acceso a la educación, y se esperaría que eso se reflejara en las opciones profesionales, pero no ha sucedido, pues se sigue presentando una división en las labores remuneradas que realizan hombres y mujeres en las oportunidades de empleo.

La inequidad de género estuvo presente en la posibilidad que se brindó a las mujeres de insertarse en algún empleo, que por tradi- 
ción han ocupado los hombres, como los puestos ejecutivos y administrativos, a pesar de haberles dado oportunidades equivalentes para desarrollar su capacidad intelectual. La misma discriminación se manifestó en los escaños parlamentarios, donde las mujeres no alcanzaron a representar ni una cuarta parte con respecto a los hombres. Pero más aún, esta desigualdad se agudiza cuando se mide la disparidad de ingresos.

Lo anterior exhibe la falta de equidad para usar las capacidades femeninas, y refleja cómo se vive en una sociedad androcéntrica, en donde las mujeres continúan siendo discriminadas y segregadas por su género, situación que se agrava cuando se habla de campesinas e indígenas.

\section{Propuestas}

La perspectiva de género se ha tratado de implementar en muchos ámbitos de las políticas públicas en México desde los años ochenta. Ello ha permitido visualizar las desigualdades sufridas por las mujeres a lo largo de la historia, y ofrecerles a ellas condiciones iguales a las de los hombres para desarrollar habilidades y capacidades tomando en cuenta las diferencias culturales en torno al género, que por lo general han sido desfavorables para las mujeres.

Logar una mayor igualdad entre los géneros es deseable, no sólo por derecho, sino también porque "produce beneficios sociales y económicos que favorecen la reducción de la pobreza" (Kabeer 2006, 32). Para el DH ha sido importante incorporar la perspectiva de género, y visualizar las diferencias entre mujeres y hombres en cuanto al acceso y control de los recursos, reconocer que ambos desempeñan papeles diferentes y tienen responsabilidades en sus propias vidas, dentro de sus familias, en su comunidad y en la sociedad, pero que esas no deben traducirse en discriminación.

La igualdad de género tiene un efecto positivo en el crecimiento económico per cápita, una vez que ha alcanzado cierto grado. La desigualdad de género en acceso al crédito, tierra, sueldos, bienes de capital y redes de comercio puede ser más relevante para el crecimiento económico de los países pobres, que las discrepancias en educación y hasta en derechos formales (Ibid., 49). 
En la región estudiada, puesto que tiene rasgos particulares, el DH alcanzado ha presentado características específicas, por lo que se propone:

- Asegurar que hombres y mujeres tengan las mismas oportunidades de ingreso, permanencia y término en la educación, así como verificar que no se promuevan estereotipos de género dentro de la educación, que denigren a las mujeres.

- Ofrecer igualdad de condiciones laborales, y establecer elementos que les ayuden a las mujeres como guarderías y prestaciones complementarias, así como salarios iguales. De tal manera que al incorporarse al trabajo asalariado no se incremente su carga en el hogar, sobre todo si se considera que en la región estudiada un gran porcentaje de los empleos remunerados está en el área de maquila y trabajo informal.

- Atender las zonas rurales o con menos habitantes, puesto que ahí las desigualdades de género son más marcadas, y las mujeres, además de sufrir discriminación por serlo, son más pobres, tienen menos servicios médicos y están expuestas a mayor violencia.

- Ajustar las políticas públicas a las necesidades de mujeres y hombres, pues a pesar de que ambos sufren discriminación y desigualdad, las condiciones y necesidades son diferentes, por lo que a las que viven en estados como Chihuahua, donde se ha reportado el mayor número de feminicidios en México, es primordial ofrecerles más seguridad pública y laboral.

- Orientar y capacitar a hombres y mujeres en temas de salud reproductiva, de tal manera que sean ellas quienes tengan el control de su cuerpo y su sexualidad, puedan decidir con quien y participar en cuántos hijos tener. Además de capacitar a ambos en la prevención de enfermedades y accidentes de acuerdo al género, ya que requieren tratamientos diferentes.

- Brindar a las mujeres acciones positivas para establecer o aumentar las opciones efectivas en la participación política y toma de decisiones.

Recibido en enero de 2010

Aceptado en junio de 2010 


\section{Bibliografía}

Abramo, Laís y María Elena Valenzuela. 2006. Inserción laboral y brechas de equidad de género en América Latina. En Trabajo decente y equidad de género en América Latina, editado por Laís Abramo, 29-62. Santiago de Chile: Organización Internacional del Trabajo (огт).

Bassols Batalla, Ángel. 1999. Investigaciones urbanas y regionales de México: ¿para conocer o transformar una realidad? En La sociedad mexicana frente al tercer milenio, coordinado por Humberto Muñoz. México: Miguel Ángel Porrúa-Coordinación de Humanidades.

BIE. 2008. Instituto Nacional de Estadística y Geografía. Banco de Información Económica. Cuentas nacionales. Producto interno bruto por entidad federativa. http://dgcnesyp.inegi.org.mx/ cgi-win/bdieintsi.exe/Consultar (12 de enero de 2010).

Birgin, Haydée. 2003. La democracia de género. Del drama individual al discurso público. En Democracia de género una propuesta inclusiva, compilado por Ángela Meentzen y Enrique Gomáriz, 40-50. El Salvador: Fundación Heinrich Bóll.

Ibarrarán, María Eugenia y Carlos Robles. 2004. Inequidad de género en desarrollo humano: el caso de México. Serie Documentos de Investigación, 12. México: Secretaría de Desarrollo Social.

INEGI. 2005. II Conteo de población y vivienda. INEGI.

2005. Marco geoestadístico municipal. http://www. inegi.gob.mx (23 de abril de 2007).

2000. Entidades federativas. http://www.inegi.gob.mx (23 de abril de 2007).

2000. XII Censo general de población y vivienda. INEGI.

1995. I Conteo de población y vivienda. INEGI. 
1990. XI Censo general de población y vivienda. INEGI.

INEGI-INMUJERES. 2005. Mujeres y hombres en México 2005. Aguascalientes: INEGI. http://www.inegi.org.mx/prod_serv/contenidos/espanol/bvinegi/productos/integracion/sociodemografico/mujeresyhombres/2005/Mujeres_y_hombres_en_Mexico_2005. pdf (25 de septiembre de 2009).

Kaaber, Naila. 2006. Lugar preponderante del género en la erradicación de la pobreza y las metas de desarrollo del milenio. México: Centro Internacional de Investigaciones para el Desarrollo, Plaza y Valdés.

Lara Flores, Sara María. 1996. Mercado de trabajo rural y organización laboral en el campo mexicano. En Neoliberalismo y organización social en el campo mexicano, coordinado por Hubert C. de Gramont, 69-112. México: Universidad Nacional Autónoma de MéxicoPlaza y Valdez.

López Barajas, María de la Paz. 2006. Indicadores de desarrollo humano y género en México. México: Desarrollo humano y género en el marco de los derechos de las mujeres, PNUD.

López-Calva, Luis F., Lourdes Rodríguez-Chamussy y Miguel Székely. 2004. Medición del desarrollo humano en México: Introducción. En La medición del desarrollo humano en México, estudios sobre desarrollo humano México, Programa de las Naciones Unidas para el Desarrollo, compilado por Luis F. López-Calva y Miguel Székely. http://www.undp. org.mx/DesarrolloHumano/serie/images/Cuadernos2003-6. pdf (22 de octubre de 2009).

Millán, Márgara. 2001. Lo oculto del currículo oculto. Reflexiones en torno al peso de lo simbólico en la vida académica. En Género, feminismo y educación superior. Una visión internacional, coordinado por Emma Zapata, Verónica Vázquez y Pilar Alberti, 305-314. México: Colegio de Postgraduados en Ciencias Agrícolas/ The British Council/ MIAC/Asociación Nacional de Universidades e Instituciones de Educación Superior. 
Nussbaum, Martha C. 1999. Sex and Social Justice. Nueva York: Oxford University Press.

PNUD. 2009. Indicadores de desarrollo humano y género en México 2000-2005. México, PNUD. http://www.undp.org.mx/IMG/pdf/ IDH_Genero_en_Mex-2000-2005.pdf (22 de octubre de 2009).

2008. Informe sobre desarrollo humano: El Salvador 2007-2008. El empleo en uno de los pueblos más trabajadores del mundo, San Salvador, PNUD. http://www.pnud.org.sv/2007/ component/option,com_docman/task,cat_view/gid, 166/Item id, 56/?mosmsg=Est\%E1+intentando+acceder+desde+un+do minio+no+autorizado.+\%28www.google.com\%29 (12 de septiembre de 2009).

2006. En la búsqueda del desarrollo humano. Presidencia del consejo de ministros, Embajada de España en Perú, Agencia Española de Cooperación Internacional. Perú: PNUD en Perú.

2002. Informe sobre desarrollo humano 2002. Profundizar la democracia en un mundo fragmentado, PNUD Programa de las Naciones Unidas. España: Ediciones Mundi-Prensa.

1995. Informe sobre desarrollo humano 1995. México: PNUD, Harla S. A. de C.V.

Programa para la Frontera Norte 2001-2006. Centro de Educación y Capacitación para el Desarrollo Sustentable, publicado 21 de octubre de 2008. http://www.semarnat.gob.mx/educacionambiental/programas/Pages/programa_FronteraNorte.aspx (26 de noviembre de 2008).

Sen, Amartya. 1997. Bienestar, justicia y mercado. Barcelona: Ediciones Paidós Ibérica/ICE/Universidad Autónoma de Barcelona.

Sen, Gita. 1996. El empoderamiento como un enfoque a la pobreza. International Development Evaluation Association (IDEAS). http://www. geolatina.net/IDEAS/documentos.php (28 de octubre de 2009). 
SEMARNAT. 2008. Programa para la Frontera Norte, Secretaría de Medio Ambiente y Recursos Naturales. http://www.semarnat.gob. $\mathrm{mx} /$ anterioredu/programas/Pages/programa_FronteraNorte. aspx (15 de octubre de 2009).

Social Watch. 2008. Índice de equidad de género 2008. Avances y retrocesos, control ciudadano. http://www.socialwatch.org/es/ avancesyRetrocesos/IEG_2008/tablas/unaescalera.htm (13 de septiembre de 2009).

Teyssier, Sophie. 1997. Sistemas financieros: un enfoque de género. Financiamiento Rural, Cuadernos Agrarios. Nueva época 15: 179-194.

Townsend, Janet. 2002. Contenido del empoderamiento: cómo entender el poder. En Las mujeres y el poder, contra el patriarcado y la pobreza, compilado por ídem., Emma Zapata Martelo, Jo Rowlands, Pilar Alberti Manzanares y Marta Mercado González, 35-65. México: Colegio de Postgraduados, Plaza y Valdez.

Zamudio Sánchez, Francisco J., Mónica G. Pérez Miranda y Carlos A. Vargas Tamez. 2001. Primer informe sobre desarrollo humano de México 1995. Guanajuato: Universidad Autónoma Chapingo y Centro de Desarrollo Humano de Guanajuato A. C. 